\title{
EL HUER'TO ESCOLAR, UNA APUESTA ECOLÓGICA PARA AFIANZAR LA ESCRI'TURA EN INGLÉS CON NIÑOS DE PRIMARIA
}

\section{THE SCHOOL GARDEN, AN ECOLOGICAL BET'TO SUPPORT THE ENGLISH WRI'TING WITH PRIMARY CHILDREN}

\section{Leidy Yazmin Camargo Aldana ${ }^{1}$ \\ Astrid Ramírez Valencia ${ }^{2}$}

Universidad Distrital Francisco José de Caldas

\section{RESUMEN}

Frecuentemente los docentes de inglés se preocupan por motivar a los estudiantes por el aprendizaje de un idioma, más el panorama que encuentran es contrario, porque estos muestran una predisposición por el aprendizaje de una lengua, también dadas por sus circunstancias socioculturales que no les da razón de ser para lograrlo, además del peso exigido por el cumplimiento de unos estándares preestablecidos en las instituciones donde laboran, sin contemplar las características ni los intereses de la comunidad educativa. Este 
artículo presenta una experiencia desarrollada en un colegio privado de la ciudad de Bogotá, donde los estudiantes tuvieron la oportunidad de crear y organizar un huerto, consiguiendo comunicar a través de escritos cortos en inglés, las vivencias experimentadas dentro de este espacio. Para la intervención se tuvo en cuenta los estudios desarrollados por autores tales como: Autum (2015), Gonzales (2018) y Mejía (2017), quienes plantean el tema del huerto escolar usada como herramienta pedagógica, al mismo tiempo, se consideró a Gonzales (2015), quien resalta la importancia del interés de quien aprende una lengua extranjera. El estudio se valió del método cualitativo y para recolectar los datos se aplicó una encuesta, unos diarios elaborados por los participantes y unas observaciones realizadas por el investigador. En la investigación se hizo un diagnóstico que dio luces para planear la intervención y posteriormente se hizo un análisis de los resultados alcanzados en esta. Para concluir, los resultados obtenidos permitieron definir que es posible que el docente investigador pueda emprender acciones pedagógicas, como en este caso, que generen conciencia frente al cuidado de la naturaleza y promuevan el desarrollo de escritos cortos en una segunda lengua, que permitan comunicar sus vivencias, dejando ver así la apropiación del idioma.

PALABRAS CLAVE: escritura, aprendizaje interdisciplinar, inglés como lengua extranjera, huerto escolar, experiencias.

\section{ABSTRACT}

Frequently the English's teachers are worried about student's motivation when they start to learn a new language, but the find view is opposite, because the students evidence a predisposition by learn a language, so they different cultural life situations, which do not establish a real reason to get it, besides the teachers have to accomplish to some predetermined standards in the institutions where they work, without to take into account the educational community 's characteristics and interests .

This article looks for to present an experience developed in a private school at the Bogota city, where the students have to the opportunity of create and organize a school garden, reporting these experiences using short English writing.

In this intervention takes into account the studies developed by author as: Autum (2015), Gonzales (2018) and Mejia (2017), who implant the school garden topic like a pedagogical tool, also it includes Gonzales (2015 who highlight the importance to learn a foreign language.

This study used the qualitative method where gather the information through an interview, some diaries made by the participants and some observations made by the researcher.

Into the investigation was done a diagnosis, which raised the intervention afterwards it presented an analysis of the achieve results.

To conclude, the achieve results allowed to define that is possible to the research teacher goes about pedagogical actions and generate conscience about the natural care and engages the second language short writing to allow it report their experiences showing a language acquisition.

KEY WORDS: writing, cross-curricular learning, English as foreign language, school garden, experiences.

\section{INTRODUCCIÓN}

Es prudente advertir que, enseñar inglés en contextos escolares colombianos de estratos populares, se ha convertido en un verdadero reto para los docentes de los colegios en Bogotá, situación que demanda de su creatividad, recursividad e ingenio para el cumplimiento de las clases, en el contexto particular donde interactúa para encauzar un propósito, en el 
caso de este escrito, con la intención de generar una conciencia ecológica; al respecto, Alonso, et al (2005) apunta que, el estudiante adquiere un conocimiento cuando le es pertinente para su interés, por esto hay que motivarlo a considerar las situaciones ecológicas, en las cuales se encuentra el planeta, de tal manera que se logre cambiar su actitud de indiferencia, encauzando sus preocupaciones, hacia el respeto por el medio ambiente que lo circunda, concientizándolo de las problemáticas de su entorno, para que sus actos puedan comenzar a dar cambios, en el contexto que se encuentra inmerso, generando así, nuevos comportamientos con los cuales sea posible la conservación del planeta para las generaciones venideras.

Creo que llegamos al núcleo del asunto donde los saberes impartidos y la experticia del profesor de inglés, deben enfrentarse a la predisposición y falta de voluntad por parte de los aprendices, requiriendo entonces la suspicacia, mente abierta y hasta la aptitud de aprender, indispensables para captar el interés como en este caso, por los temas relacionados con el ambiente ecológico, de tal manera que la enseñanza se encamine en esta causa, o por aquellos temas de relevancia para la actualidad.

En búsqueda de una respuesta a esta exigencia y en aras de encontrar alternativas frente a esta situación se hizo un sondeo a los niños de quinto (5to) de primaria, por presentar la situación anteriormente explicada, permitiendo detectar los siguientes indicios: primero, tenían poco interés por aprender, de allí su baja pretensión de participar en las actividades propuestas en la clase de inglés, segundo, se sentían obligados a desarrollar las actividades propuestas por el profesor de lengua, por tal razón su avance en esta clase, era escaso, Por último, manifestaron que por el contrario, estaban interesados en desarrollar actividades relacionadas con temas ecológicos.
A causa de ello y con estos hechos en ciernes, la investigadora, acompañada de su condición de docente, decide planear diversas estrategias, que permitieran resolver estas inquietudes, tratando de identificar alternativas, para sopesar los inconvenientes detectados en el sondeo, teniendo en cuenta la situación del contexto en el cual se circunscribe el mundo actual, se decidió abordar el tema ecológico, por considerar que involucra algunas de las otras incidencias, identificadas en ese tanteo.

Unavezidentificadaesta temáticay considerando que existe una necesidad marcada, de generar conciencia frente al cuidado de la naturaleza, a pesar de los hábitos que acompañan nuestro diario vivir, razón justificada para establecer una interconexión entre el aprendizaje de la lengua inglesa y las temáticas desarrolladas en la ecología y las ciencias, lográndose converger un propósito común, encauzado hacia el desarrollo de un huerto escolar.

En búsqueda de una respuesta a esta exigencia se acude a Sántiz (2018) quien bosqueja la creación de los huertos escolares con una mirada pedagógica, considerándolos un laboratorio natural, dentro del cual interactuar los estudiantes involucrándose en experiencias que realmente les interesa, convirtiéndose así en un triple pretexto, el primero, que permite desarrollar una práctica vivencial generadora de cambios en el ambiente cotidiano y en las interrelaciones del salón de clase, el segundo, consiste en crear conciencia sobre el uso, cuidado y conservación de los recursos naturales, y el tercero y último, involucra el desarrollo de habilidades del idioma, el cual para este caso particular, se esboza en el afianzamiento de la escritura en la lengua extranjera, con un grupo de niños.

Lo que conduce a advertir que la creación de este huerto, viabiliza el desarrollo de otras habilidades, dando paso a la inclusión e interdisciplinariedad en aras del desarrollo de competencias relacionadas en este caso con 
el saber del idioma inglés, tal como lo plantea CIGA Colecciones (2014) este tipo de iniciativas dan apertura al fomento de un ciudadano autónomo, solidario y armonioso con el medio ambiente, que responde a las necesidades de la vida cotidiana, además de estar adquiriendo un saber relacionado con la lengua extranjera.

Esta salvedad se ratifica, con los argumentos de Moreira (2017) y Ausubel (2002) quienes manifiestan que la adquisición de nuevos conocimientos, son efectivos siempre $y$ cuando sean importantes para quien aprende, expectativa que promueve el desarrollo de la comprensión, la criticidad, la explicación, el cuestionamiento y la argumentación, dando tránsito a la búsqueda de soluciones, frente a las distintas situaciones que lo impacten, incluso dentro de su vida personal.

Se han discutido hasta ahora, diferentes elementos relacionados con el cruce de diversos campos del conocimiento, Asimismo, se reconoce la condición de considerar el tema de la habilidad de la escritura, para lo cual se aborda a Larios R., \& Rodríguez (2018) quienes destacan la importancia de esta habilidad, no solamente por el momento escolar vivido por el estudiante, sino por ser un proceso que lo acompaña durante toda su vida, que le permite además comunicar ideas y experiencias, con las cuales también surge la necesidad y pasión por el ejercicio de la escritura, siendo este, un indicio de apropiación de la lengua extranjera.

Para ello, este artículo de investigación incluye, converge y pone en diálogo diferentes posturas teóricas desarrolladas y de manera particular acude a González (2015) quien confirma esta consideración, complementando que la producción escrita es una habilidad compleja, porque allí convergen diferentes conocimientos básicos de la lengua, además porque refleja el dominio del idioma.
Por otro lado, este escrito orienta su mirada hacia el MEN (Ministerio de Educación Nacional), (2006) entidad que enfatiza la necesidad de generar en el estudiante la capacidad de la creación de textos cortos, relacionados con su entorno, con la producción de pequeñas historias, que reflejen el dominio de esta habilidad importante para el individuo, llegando incluso a ser considerada en las pruebas nacionales e internacionales.

En esta revisión documental se revisaron los conceptos manejados por los estudiantes de grado quinto, fundamentados en el conocimiento correspondiente al área de biología, en relación con las plantas, sus características y calificaciones particulares, sin embargo, en esta área no se tomaba en cuenta la ecología humana, MacMeekin, M. (2013) y Williams y Dixon (2013) consideran fundamental esta área por posibilitar la generación de conciencia y responsabilidad que tiene el ser humano, frente a la afectación que se está dando a la naturaleza.

A partir de estas premisas teórico-conceptuales, el investigador se motiva y prepara para promover y estimular el sentido proteccionista al medio natural por parte de los participantes, de tal manera, que se genera una conciencia sustentable que busque armonizar la relación entre este y el planeta. Esto motivado por la responsabilidad de la escuela, para cultivar conciencia en los pequeños, quienes de esta forma interiorizan la importancia de sostener un medio ambiente amigable, que permita la supervivencia a las generaciones futuras.

De esta manera se lleva a ser conscientes, que somos parte de un ecosistema que hace parte de sistemas complejos, dentro de los cuales coexisten variados elementos interconectados, donde los humanos están acompañados de unidades bióticas y abióticas, de tal manera que unos dependen de los otros, sin embargo, se caracterizan por ser autónomos, pero conviven 
en un marco de múltiples relaciones, que permiten desiguales modos de existencia.

Esta consideración lleva a pensar en la forma en que los profesores proyectan a los estudiantes su relación con el ecosistema, de aquí nuestra gran responsabilidad para que, desde ya, estos niños en edad escolar creen unas condiciones ambientales diferentes para el futuro, las cuales permitan conservar la vegetación, promoviendo la subsistencia del medio ambiente.

Hay mucho que decir sobre esto, Scott, R. (2017), determina que existe la necesidad de buscar maneras para modificar el consumo de los recursos, esto porque si no se emprenden acciones inmediatas surgidas al interior de la escuela, en pocos años es probable que las generaciones futuras, no disfruten de los ecosistemas existentes en la actualidad, que mejor circunstancia, para que los docentes, empiecen a emprender acciones en compañía de los estudiantes, constructoras de alternativas que propendan por la protección del medio ambiente.

Por tal motivo, los caminos y constructos teóricos explorados hasta aquí conducen a emprender diversas acciones con las cuales se alcance este objetivo, de allí la necesidad de plantear la creación de un huerto que permita establecer una conexión real con las situaciones ecológicas del momento, incentivando al mismo tiempo, la creación de hábitos saludables para el ecosistema y para la vida de este estudiante, de tal manera, que se logre una aprehensión del conocimiento de la lengua, esta será la excusa para la implementación y el desarrollo de experiencias, de tal modo que sus opiniones e ideas respecto al desarrollo de la Huerta escolar, quedaron plasmadas en diversos escritos, creados en lengua extranjera.

Este enfoque también es abordado por Gonzales (2018) quien explica que la implementación de un huerto escolar permite sembrar, cultivar y recolectar hortalizas y verduras, pero para organizarlo, se necesita de una zona para la siembra, que requieren unos compartimentos, cajas, así como algunas herramientas con las cuales se puedan sacar los frutos de la tierra, dando la alternativa también de ser sembrado como un huerto vertical en botellas plásticas, que en los tiempos actuales es bastante común, especialmente por la falta de espacio, en las diferentes instituciones escolares.

Esta visión permite estipular que es, inevitable la necesidad de ir más allá de la construcción de un huerto escolar, pues no se trata de habilitar una zona idónea para un cultivo, sino por el contrario, se busca involucrar al estudiante en la sensibilización frente al cuidado del planeta y su función armónica con él, con lo que se pretende, tener repercusiones positivas en los educandos, yendo más allá de la escuela, generando conciencia por la conservación del medio ambiente, esto porque la humanidad está llevando a un desgaste ambiental constante del planeta, por ello, la escuela debe emprender acciones que promuevan el cuidado, el buen manejo y el desarrollo sostenible de los recursos naturales, donde sólo el ser humano, podría ser el eje dinamizador de este cambio.

A partir de las premisas teórico-conceptuales, se traza un referente relacionado con este tema a través de Morgan (2012) quien desarrolló un proyecto de investigación concerniente con el afianzamiento de los conceptos del área de la biología en inglés, donde fue posible la apropiación del vocabulario, así como las nociones relacionadas con las plantas y su cultivo, que posteriormente, facilitaron la explicación de esta experiencia.

Continuando con este recorrido teórico, encontramos que Feito, (2011) Haros et al (2013) y Mejía (2017) entre otros que focalizan su mirada investigativa en el uso de las huertas escolares usadas como herramienta de aprendizaje ambiental, haciendo un acercamiento de los 
estudiantes a este espacio, con el propósito de hacer un seguimiento sobre, el cuidado de las plantas, tomando en cuenta los conocimientos previos del niño, sobre con este tema.

Como resultado, se logró una conexión de los estudiantes y la comunidad educativa para alcanzar este propósito, de igual manera, se mejoró la toma de conciencia ecológica, percibida mediante el respeto y el amor propio, promoviendo dinámicas de participación y cooperación entre ellos, con los cuales fue posible la identificación de algunos problemas locales, relacionados con el medio ambiente que involucran la colaboración de la comunidad educativa, en la consecución de este logro, llevándolos así para que fueran los mayores protagonistas de la protección al planeta.

\section{METODOLOGÍA}

La investigación como proceso de la indagación del conocimiento, busca dar respuesta a una problemática, para alcanzar este propósito se apoya en el uso de métodos científicos.

Afin de dar cuenta de ellos, Cook, Ty Reichardt, C. (1979), Cadena et al (2017) ratifican la existencia de dos métodos, el cualitativo y el cuantitativo usados en el análisis de la información recogida en el marco de una investigación.

Aceptada la existencia de estos métodos, el cuantitativo analiza el problema en términos numéricos, arrojando datos caracterizados por la precisión basada en el procedimiento de la medición del dato, mientras, el cualitativo describe situaciones, eventos, comportamientos, observaciones y estudios de caso prácticos, teniendo en cuenta la conceptualización y la aportación basada en la mirada del investigador que comprende, analiza y describe los hallazgos; este proyecto se enfocó en el método cualitativo, interpretativo, donde fue primordial la óptica y la voz del investigador.
Para desarrollar el presente estudio se utilizaron algunas observaciones las cuales según la opinión de Vela, F. (2001) permitió reflexionar frente a las acciones emprendidas por el investigador, de tal manera que se hizo un seguimiento al proceso llevado a cabo dentro del marco de la intervención, asumida aquí por el profesor de inglés, quien hace las veces de investigador, de tal manera que en este instrumento quedó registrada la voz del indagador, frente a las acciones desarrolladas en su condición de profesor de inglés, con un grupo de estudiantes del grado quinto.

De igual manera, se utilizaron unos diarios de campo donde quedaron inscritas las voces de los estudiantes, es decir, los participantes de esta experiencia, dándole énfasis al conocimiento adquirido durante el desarrollo de esta intervención, que incluye diferentes etapas.

Estos registros hechos por los estudiantes participantes permitieron el ajuste de nuevas actividades propuestas por el docente investigador, las cuales buscaban generar un ambiente mucho más agradable, para la organización del huerto, estableciendo así, una conexión de empatía entre el investigador y las experiencias de los estudiantes, que quedaron registradas en los diarios escritos, en lengua inglesa.

De igual manera se aplicaron entrevistas informales a algunos estudiantes al azar, debido a que, a pesar de reconocer la importancia del idioma, justificaban su desinterés con expresiones como "eso es muy difícil" o "yo nunca entiendo".

El objetivo de este estudio fue describir cómo los estudiantes tomaron conciencia frente al cuidado del medio ambiente, con la intención de emprender acciones, con las cuales se velará por la conservación de la naturaleza, además de sensibilizarlos, de tal modo que tomaran la iniciativa de plasmarlo en un escrito, promoviendo 
así la expresión de estas experiencias en forma escrita, en inglés.

Este proceso fue desarrollado en 12 sesiones que comprenden la etapa de exploración del sitio de aplicación de la propuesta, así como un diagnóstico que sirvió de insumo para la intervención, también se organizaron algunas jornadas de sensibilización, para luego llevar a cabo la implementación de la propuesta, finalmente, se hizo un análisis de los hallazgos, identificados en esta experiencia.

Para lleva a la marcha esta propuesta se contó con el espacio de la clase de inglés, dentro del cual, el investigador- profesor planeó actividades alrededor del tema de la huerta, de manera simultánea se incluyó temáticas de inglés, correspondientes al currículo establecido por el colegio, que, contemplaba su identificación en el espacio del mundo, en el país, en su hogar , en la escuela y por último, dentro del huerto escolar, llevándolo a descubrir, que está llamado a ser un cuidador del planeta.

\section{PARTICIPANTES Y CONTEXTO}

El Proyecto fue desarrollado en un colegio privado mixto de la localidad quinta (5) de Usme de la ciudad de Bogotá, cuyos estudiantes son de estrato socioeconómico de nivel 2, los participantes correspondientes a dos grupos de estudiantes del grado quinto, donde $40 \%$ eran niñas y $60 \%$ eran niños, tal como se aprecia en la gráfica 1, sus edades oscilaban entre 11 y 14 años.

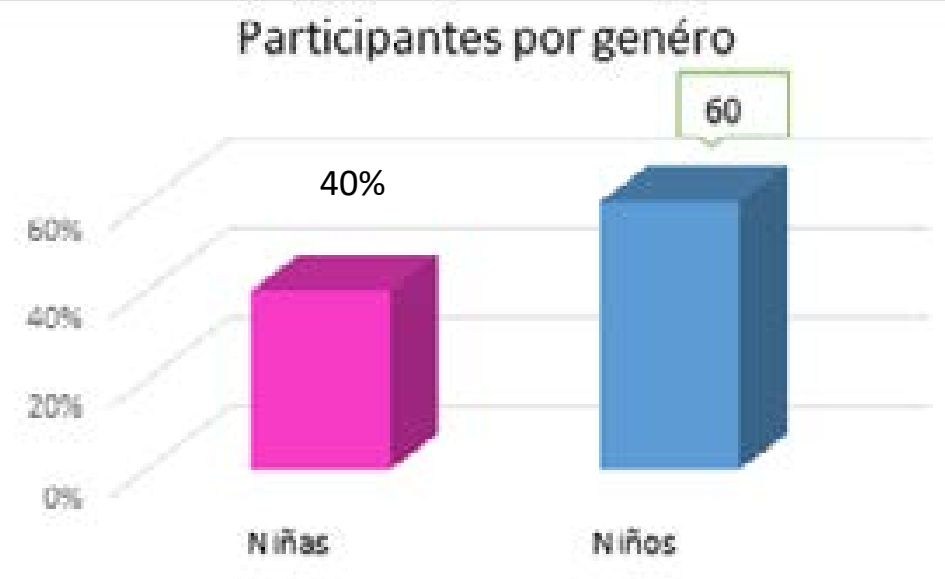

Gráfica 1. Participación por género fuente: propia.

Una vez hecha la intervención se pudo apreciar un trabajo homogéneo demostrado en el mismo nivel de progreso de los participantes, es decir, su desempeño fue homogéneo, indicando que el género, no fue un factor decisivo dentro de esta investigación.

Con esto cabe agregar que como parte del diagnóstico se incluyó el registro de una aproximación a los factores correspondientes a primero, la lengua - vocabulario, segundo, el huerto y el medio ambiente, tercero, al contexto y finalmente el cuarto fue la escritura en inglés, los cuales hicieron parte de la etapa inicial de este proyecto.

\section{DIAGNÓSTICO}

En esta etapa, se hizo una exploración a las diferentes fuentes de indagación, con el propósito de identificar el problema y sus posibles afectaciones o tensiones.

En este sentido esta etapa no sólo fue descriptiva, sino que, por el contrario, sirvió de fuente dé explicación y pronóstico, frente a las tensiones alrededor de las cuales, se estableció esta investigación. 


\section{LENGUA Y VOCABULARIO}

Comencemos diciendo que la institución no contaba con recursos tecnológicos e informáticos idóneos, y además había problemas de conectividad, en algunas áreas de la institución.

Agregando a lo anterior, se hizo una revisión de los planes de estudios, donde se constató que los contenidos focalizaban aspectos gramaticales, basados esencialmente en el diligenciamiento de libro guía, probablemente por ello, la mayoría de los participantes habían perdido el interés en aprender la lengua extranjera, pues esta situación los hacía percibirla, como una obligación.
Para definir el nivel de dominio de la lengua y de vocabulario de la lengua inglesa se aplicó un cuestionario inicial, dentro del cual, se evaluó el dominio de algunas estructuras básicas y el conocimiento del vocabulario relacionado con el huerto, observándose entre otros, los siguientes resultados, solamente el $20 \%$ pudieron resolverlo de manera adecuada, el $10 \%$ de ellos decidió simplemente colorear y no resolver las preguntas planteadas, mientras el $70 \%$ no comprendió el contenido de los enunciados, correspondientes a las preguntas, estos resultados se evidencian en la gráfica 2, para el caso de los estudiantes que tuvieron muchas dificultades, la docente hizo un acompañamiento para intentar resolver las deficiencias detectadas.

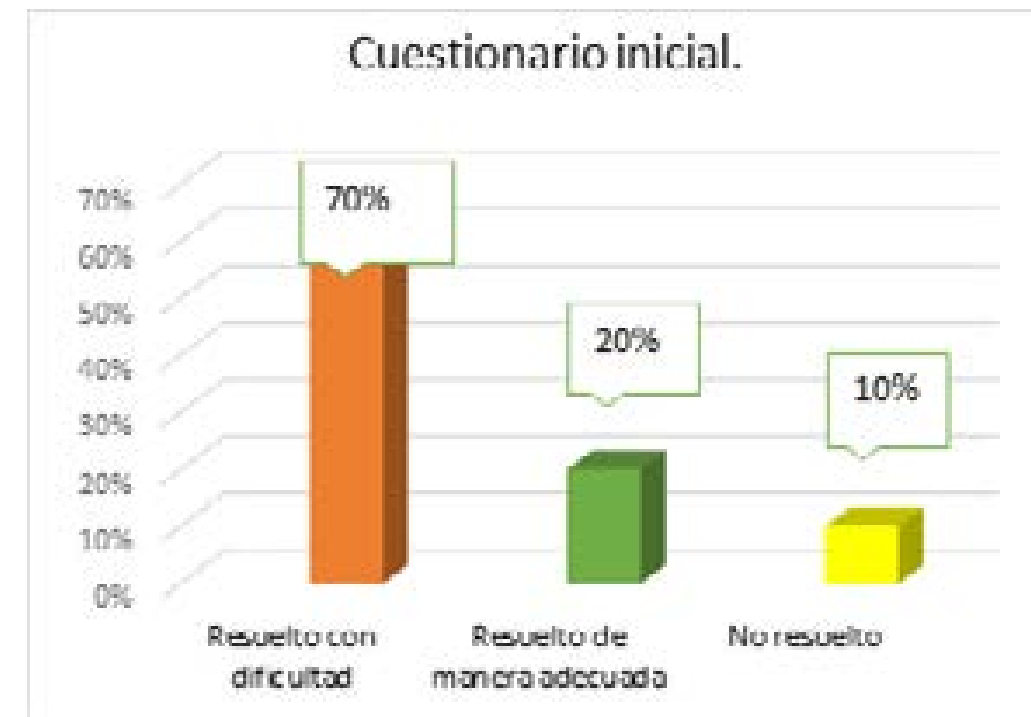

Gráfica 2. Solución de cuestionario inicial. Fuente: propia

\section{EL HUERTO Y EL MEDIO AMBIENTE}

Una vez tomada la decisión de organizar un huerto se optó por seleccionar el espacio dentro del colegio donde se instalaría, el cual surge como una alternativa viable, pretexto para experimentar y aprender inglés de una manera dinámica, favorable e interesante; al comienzo, se organizó una actividad en forma de nave espacial, relacionada con la presentación del personaje Blue, para hacerla más creativa, tal como se ve en la foto 1
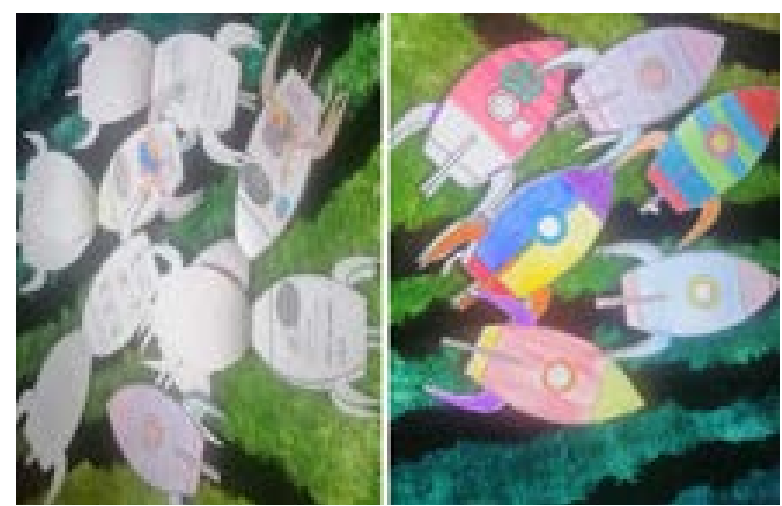

Foto 1. Presentación de la actividad del huerto en forma de nave espacial. Fuente: elaboración propia 


\section{EL CONTEXTO}

El colegio no contaba con un espacio para la creación del huerto, por tal razón se implementó la construcción de un huerto vertical, en botellas plásticas y algunas cajas de madera, donde se sembraron algunas plantas aromáticas, y alimenticias como fresa y tomate cherry, considerando la sabiduría de los conceptos de la botánica, la agricultura y la ecología desde la visión humana, en los estudiantes, tal como se observa en la foto 2 .
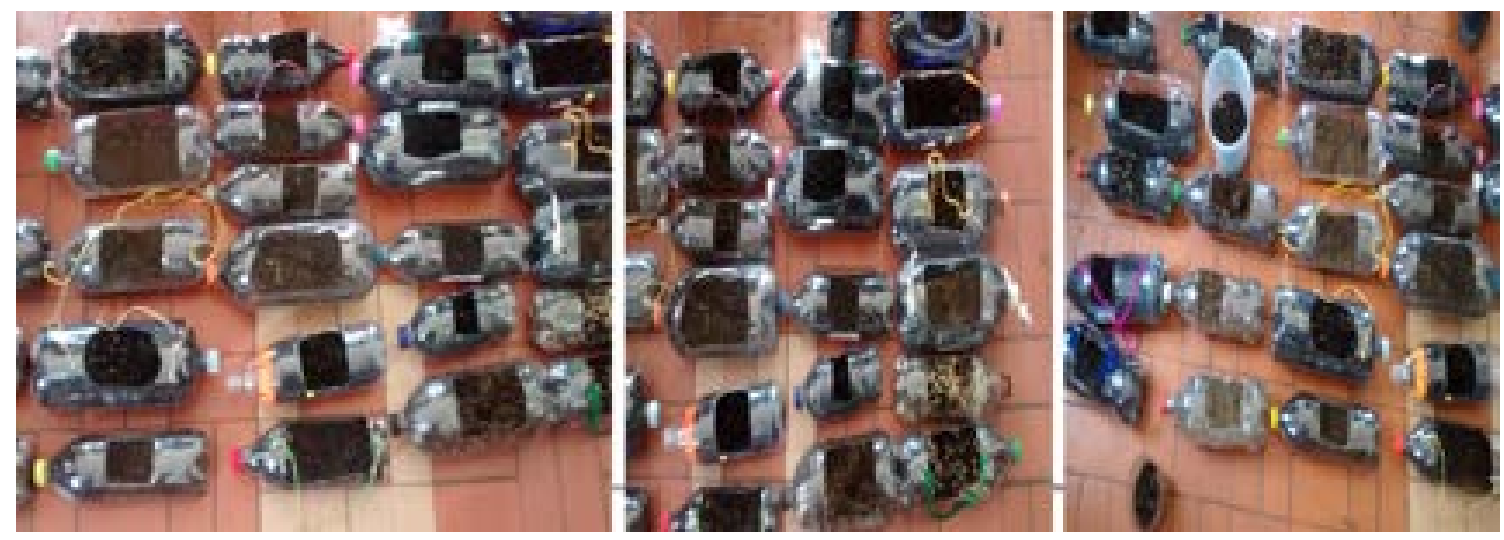

Foto 2. Presentación de la creación inicial del huerto. Fuente: elaboración propia

\section{LA ESCRITURA EN LA LENGUA EXTRANJERA}

Recordando que una etapa de apropiación del idioma extranjero está relacionada con el proceso escritural, se creó la necesidad de expresar la experiencia del huerto de esta forma, para ello, se hacían descripciones simples tomado como base las observaciones de los participantes, quienes expresaban cómo se sentían al actuar en las actividades del huerto, desarrollando así, el proceso de escritura de manera lenta, pero progresiva; para ello comenzaron por elaborar oraciones simple, luego frases compuestas, para llegar finalmente, a la construcción de párrafos cortos, resaltando la gran sensibilidad por parte de los estudiantes, sin darle demasiada importancia al rigor gramatical, ni a la corrección, para motivar su participación y su impulso por plasmar su pensamiento, en la lengua extranjera.

Conviene observar que esta actividad por el contrario se centró, en el desarrollo integral y la promulgación de la ecológica humana, impulsando un balance entre el manejo armonioso de la naturaleza y el ser, en la búsqueda de la conservación del medio ambiente, mediante el análisis de su comportamiento específicamente, cuando se encontraban involucrados en la creación del huerto, promoviendo el rescate de los valores éticos universales, que diera cabida a procesos de reflexión encaminados a la conservación del medio ambiente, pretendiendo controlar con ello, el deterioro del entorno, buscando el bienestar de sus integrantes, haciendo que fueran conscientes de la importancia de este tipo de actividades, para que en un futuro la llevaran, incluso a sus hogares.

De esta forma se procuró extender esta experiencia en su hábitat cotidiano, alcanzando con ello un desarrollo de integración a la sociedad, empleando como instrumento de pensamiento la posibilidad de organizar, sistematizar y expresar ideas, sentimientos y deseos, para ser convertidos en una mediación en la adquisición del conocimientos en todas las demás áreas del saber, para así exteriorizar sus ideas y conceptos, producto de esta 
experiencia, para luego, sentir la necesidad de, ser plasmados en forma escrita, y finalmente, con apoyo del docente se hacían los ajustes al texto escrito, para más adelante, ser expuestos dentro de la comunidad.

\section{INTERVENCIÓN PEDAGÓGICA}

Esta intervención pedagógica se diseñó en tres etapas que a continuación se enuncian.

\section{Primera}

Para esta sesión se organizó un tour por los espacios del colegio, identificando en inglés los diferentes sitios, familiarizando a los participantes con este vocabulario en inglés.

\section{Segunda}

Aquí, los estudiantes crearon situaciones relacionadas con su colegio, así como con los miembros de su familia, describiéndolas en inglés, para lo cual utilizaron algunos adjetivos que combinados con algunas estructuras básicas y con el vocabulario visto en clase, permitió a la investigadora impartir una charla, explicando la importancia de la utilización de un huerto escolar. Ver foto 3
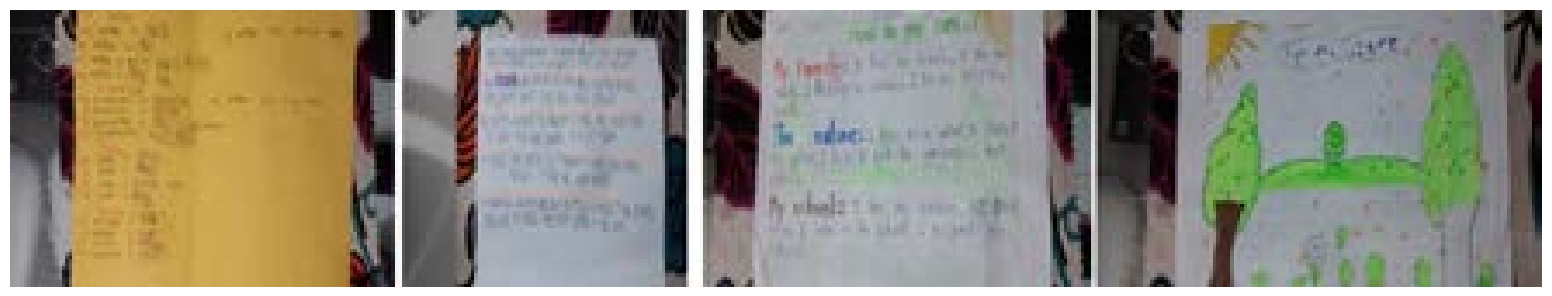

Foto 3. Muestra de textos construidos por los participantes. Fuente: propia

\section{Tercera}

En esta etapa, los participantes crearon pósters, resaltando la importancia del medio ambiente, generando conciencia en la comunidad acerca de la conservación del huerto escolar, con lo cual se buscó invitarlos a integrarse, en este proyecto.

En lo relacionado con el proceso escritural se evidenció falencias en cuanto a la gramática y el escaso vocabulario, sin embargo, esto demostró la realidad de su proceso de aprendizaje del idioma inglés, así como su ambientación y percepción, frente a los problemas ambientales.

Dentro de los procesos de escritura, se logró detectar errores gramaticales y otros relacionados con el uso de vocabulario específico, pero en esta parte de la investigación, estos desaciertos fueron irrelevantes, porque lo más importante era que los estudiantes lograran confianza en sus procesos escriturales, para poder comunicar sus ideas y percepciones sobre los problemas ambientales, en inglés, lo cual se puede apreciar en la foto 4 .

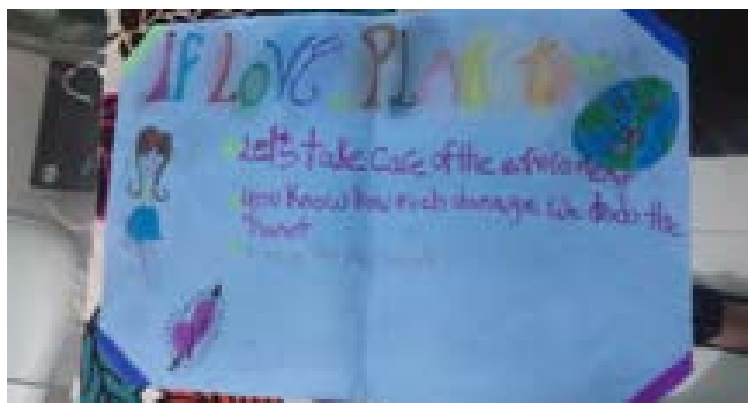

Foto 4. Muestra de posters hecho por los participantes. Fuente: propia

\section{Cuarta}

Esta etapa fue usada para evaluar la propuesta, buscando analizar el nivel de participación de los estudiantes dentro de la experiencia de organización del huerto escolar, en cuanto a los textos escritos en inglés se evidenció un progreso en el manejo y uso de los aspectos gramaticales 
y de vocabulario, donde el investigador estuvo atento a ofrecer una oportuna retroalimentación, los involucrados demostraron entusiasmo e interés en el ejercicio de corrección con el interés de exponer su trabajo de forma impecable y con orgullo por el deber cumplido.
Finalmente, el éxito de los diarios de campo elaborado por los participantes dio su fruto en los posters, donde quedó plasmado el proceso y el resultado final de la experiencia, como se observa en la foto 5 .

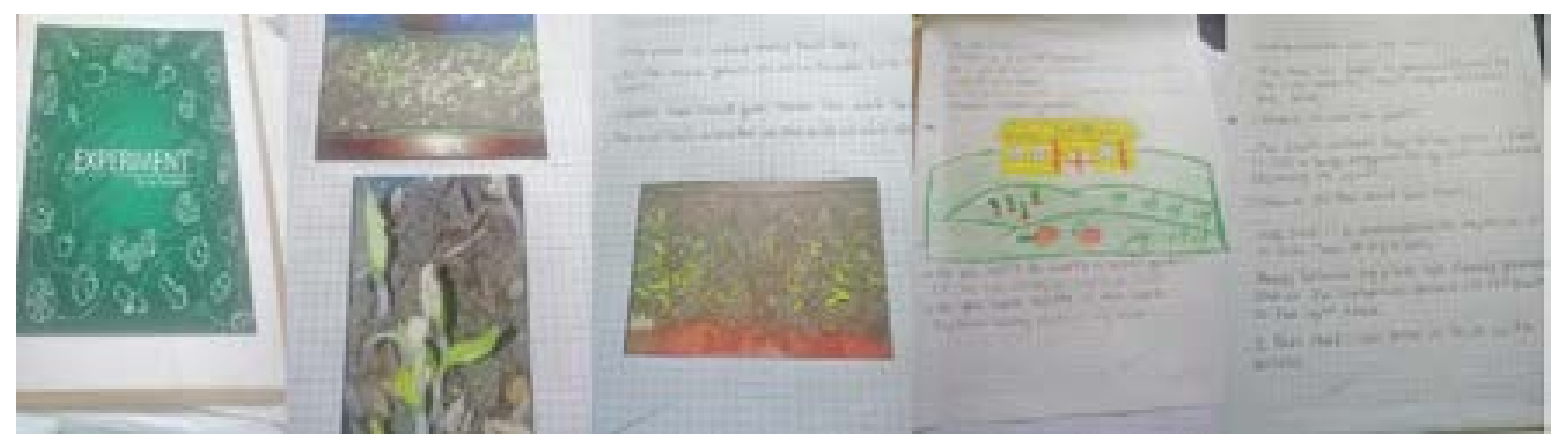

Foto 5. Foto del diario, así como de los posters producidos por los niños como resultado final de la experiencia. Fuente: propia

\section{INTERPRETACIÓN}

La creación del huerto escolar no es tarea fácil, como bien lo plantean los autores Gonzales, M. (2018) y Mejía (2017), pero apoyándose en esta actividad como estrategia pedagógica es posible la creación de un espacio que logre cambiar la percepción de muchos estudiantes sobre sus clases de inglés, se puede afirmar que en ellos se logró despertar un interés ecológico y humano y un deseo de mejorar su dominio de la lengua inglesa, de tal manera que estuvieron expectantes, cada vez que se les indicaba que la clase se realizaría en el huerto, surgían preguntas entre ellos como: -What do you find in it?, Are there any product?

Respecto a la lengua inglesa, se logró el dominio de estructuras que dieron acceso a la comunicación a través de la construcción de párrafos escritos, donde se pudo apreciar el aumento de su vocabulario y el uso adecuado de la gramática en la lengua inglesa apoyados en los postulados de Gonzales (2015) quien explica que en la producción de textos escritos convergen diferentes conocimientos del idioma
Creo haber mostrado que el huerto fue el hilo conductor para adentrarse en el empoderamiento del manejo de la estructura gramatical del idioma extranjero y la apropiación espontánea y emotiva por la conservación del medio ambiente, para lo CIGA Colecciones (2014) como también Sántiz (2018) por explicar que esta temática puede ser usada para impartir diversos saberes, en este caso, de agregado estaba la expectativa de recoger el fruto de su cosecha, pensando en la alimentación correspondiente a lo personal y al esfuerzo de lo cultivado, en el huerto escolar.

Respecto a la participación, hubo un marcado incremento porcentual en comparación con los índices obtenido antes de la intervención, como lo señala Gonzales (2015) es vital la participación en el aprendizaje de un idioma, a pesar de esto, la gráfica 3 , demostró una intención por participar baja, con un $70 \%$, sin embargo, luego de la intervención por parte del investigador, y al mismo tiempo docente en formación, se pudo detectar que desde el momento en que se inició la intervención la participación presentó un avance significativo llegando al $85 \%$ de incremento, lo que de una u 
otra manera evidencia, que el trabajo producto de esta investigación es pertinente para apropiarse del idioma extranjero. (Ver gráfica 3)

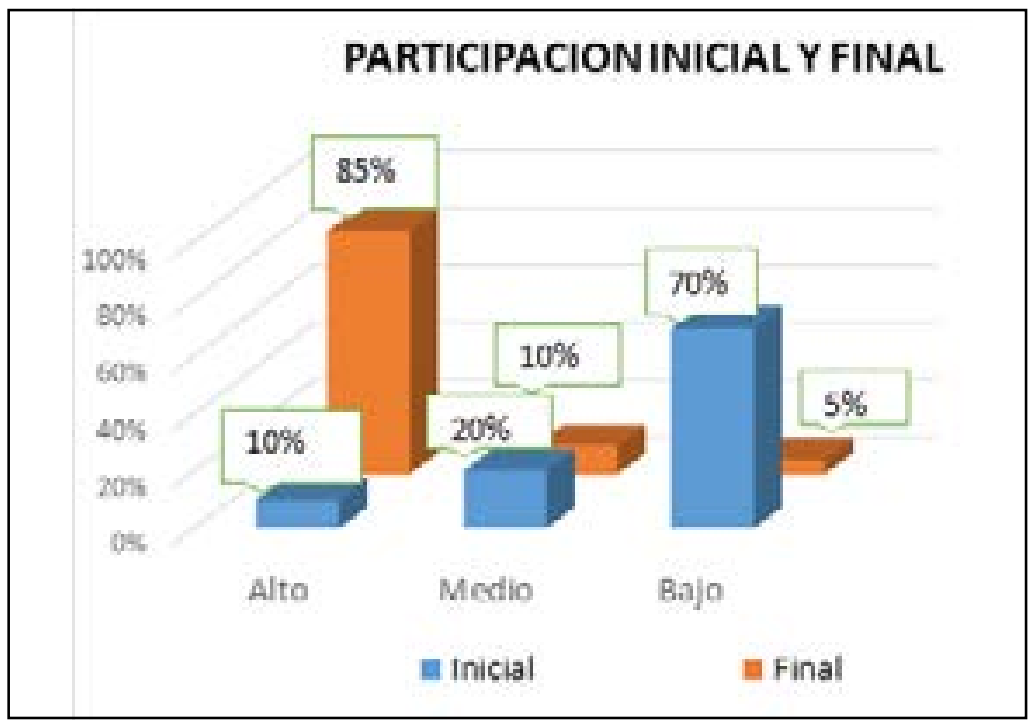

Gráfica 3. Participación inicial y final en la clase de inglés. Fuente: propia.

\section{PRODUCCIÓN DE TEXTOS}

Por otra parte, esta investigación arrojó como efecto, que antes de la intervención solo el $10 \%$ de los participantes construyeron textos con coherencia y cohesión, a pesar que el MEN (Ministerio de Educación Nacional), (2006) considera pertinente la producción de texto, situación superada porque, luego de la intervención, los hallazgos fueron contundentes, pues el $85 \%$ lograron expresar sus ideas en forma escrita en la lengua extranjera, produciendo cortos escritos, relacionados con el tema del huerto, agregando la aptitud ecológica humana, en la cual primó la solidaridad mediante el emprendimiento de acciones que buscaban entrar en armonía con el medio ambiente, mostrando una actitud resiliente, proactiva y conciliadora con sus congéneres; estos resultados se muestran en la gráfica 4 .

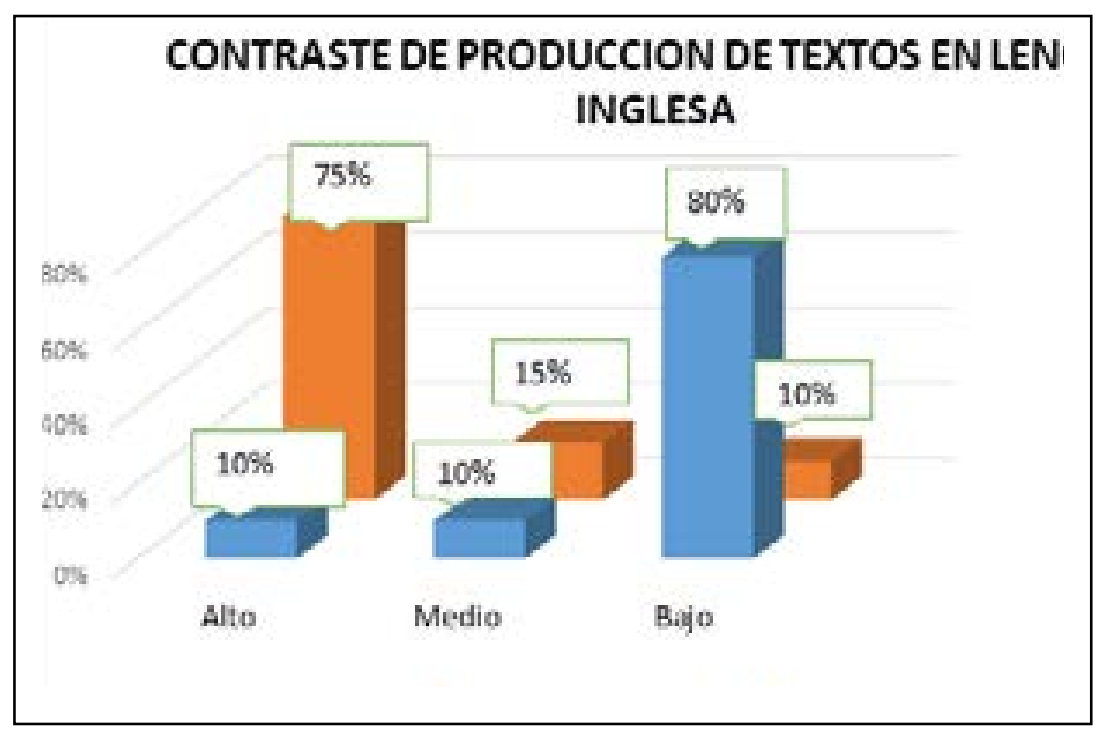

Gráfica 4. Contraste de escritos generados antes y después de la intervención. Fuente: propia 
Para terminar, Morgan et al. (2010) y Bucher (2017) en sus trabajos concibieron de manera acertada la creación de huertos escolares como un espacio apropiado para adquirir nuevos conocimientos, generando procesos vivenciales, desencadenando acciones que le den sentido a la condición humana, en pos de promover la conservación del planeta, basado en el rescate de valores éticos como la solidaridad, la igualdad, la justicia y la equidad.

Bien pareciera por todo lo anterior que, todos estos rasgos positivos buscaban llevar a los participantes a sentar una conexión con su universo, considerando a autores como Feito, (2011) Haros et al (2013) y Mejía (2017) quienes plantean la necesidad de generar un contexto basado en la orientación y conservación del ambiente, con optimismo y espiritualidad, dándoles la oportunidad de proyectarse a sí mismos, como seres defensores de un planeta para el futuro, comprendiendo al mismo tiempo, la fuerza de su trascendencia, para emprender acciones como las de la creación del huerto escolar que le dan una dimensión directa y de compromiso con el contexto circundante, mediante el emprendimiento de sus acciones en pos de la conservación y protección, para el futuro.

De otro lado, se buscó que los participantes se apropiaron de su proceso de aprendizaje, tomando las indicaciones de Rivera, (2004) que permiten incentivar la consolidación de conocimientos del investigador - profesor, quien tuvo que ampliar su conocimiento respecto al manejo, sostenimiento y creación de un cultivo, así como el reconocimiento de vocabulario en inglés relacionado con las plantas, con la forma de cultivar y con la organización del huerto y las plantas alimentarias, al igual que con los frutos que se cultivaron, lo cual permitió fortalecer el conocimiento y explorar otras áreas consiguiendo apropiarse de este vocabulario en el profesor, para estar en capacidad de fortalecerlo en los pupilos.

\section{CONCLUSIONES}

Teniendo en cuenta los procesos evidenciados en investigaciones preliminares como las de Morgan (2012), Moreira (2017), Mejía (2017), Buchet (2017) y Gonzales (2018) donde se utiliza el huerto escolar como herramienta pedagógica, se logró comprobar a través de las observaciones y los diarios creados por los estudiantes, que el uso del huerto escolar permite generar en los estudiantes la motivación, que abre paso a su interés por observar,aprender, y también a aumentar su capacidad de producir textos escritos, en el idioma inglés.

Por otra parte, se logró fomentar en los estudiantes el deseo de compartir el espacio del huerto escolar con la comunidad, donde ellos como agentes activos daban a conocer su importancia, empoderándose así, de un despertar consciente frente al cuidado de los recursos naturales, dando paso de este modo a proponer futuras investigaciones relacionadas con este tema de vital importancia, como es el cuidado de los recursos naturales.

La idea es, no desconocer que el aprendizaje interdisciplinar se vuelve significativo y es una gran opción para motivar y enseñar a los estudiantes de primaria, por dar una nueva perspectiva a la enseñanza del inglés dentro de una institución educativa de estrato bajo, dando cabida a la enseñanza de una lengua extranjera, relacionándola con un contexto cercano, vivencial y con un propósito específico para los estudiantes, que despiertan su interés.

Con respecto al docente, se requiere que tenga aptitud, compromiso para ser promotor de la creatividad, la innovación, la dedicación demostrando tener vocación de explorador, porque se sabe que la docencia no es una labor sencilla, por lo tanto, el docente debe 
mantenerse con el deseo de capacitarse y seguir aprendiendo cada vez más, de tal manera que logre establecer nuevos propósitos de enseñanza, llamativos e interesantes para los aprendices.

\section{REFERENCIAS BIBLIOGRÁFICAS}

Alonso, C. y. (2005). Estilos de aprendizaje. Obtenido de http://estilosdeaprendizaje. es/menuprinc2.htm

Alvares, B. G. (2010). La tierra viva: Manual de agricultura Ecológica. Puerto Rico.: Coqui Santiago,.

Ausubel, D. P. (2002). Adquisición y retención del conocimiento. Una perspectiva cognitiva. . Barcelona.: Ed. Paidós.

Autum, C.(2015).InfluentialEnvironments:School garden impacting Arizona .

Ballester, A. .. (2002 ). "El aprendizaje significativo en la practica.Cómo hacer el aprendizaje significativo en el aula". Obtenido de Obtenido de www. pensamientoestrategico.com.

Berenice Larios R., \&. E. (2018). Teorías del aprendizaje. Bogota: Editorial magisterio. .

Bringel, B. (2015). Fronteiras difusas: movimentos sociais, intelectuais e construções de conhecimen-tos". In: Marco Antonio Perruso e Mônica da Silva Araújo (Orgs.) . Ciência e Política: memórias de inte-lectuais. , 57-69.

Bucher, K. (2017). Opening garden gates: Teachers making meaning of school gardens in Havana and Philadelphia. Teaching and Teacher Education, 63, 12-21. doi: 10.1016/j.tate.2016.12.003. Obtenido de doi: 10.1016/j. tate.2016.12.003
Cadena Iñiguez, P. (2017). Cadena Iñiguez, P., Rendón Medel , R., Aguilar Avila, J., Salinas Cruz , Métodos cuantitativos, métodos cualitativos o su combinaciónen la Investigación:Un acercamiento en las ciencias sociales. Revista Mexicana de Ciencias Agricolas, pp.1603-1617.

Carrizosa Umaña, J. (s.f.). "Evolución del concepto de desarrollo sostenible", en Globalización, medio ambiente $y$ desarrollo. . Medellín: Medellín: Fondo Editorial Cancillería de San Carlos/ Penca de Sábila.

Colecciones, C. (s.f.). Regnum hominis. Prolegómenos para una antropología filosófica de la crisis ambiental. . México: México: UV/UNAM-CIGA.

Colmenares, M. ,.-1. (2012). Investigación Acción Participativa: Una metodología Integradora del Conocimiento y la acción Voces y Silencios. . Revista Latinoamericana de Educación , ISSN-e 2215-8421, Vol. 3, №.1, pp.102-115.

Cook, T. (1979). Qualitative and quantitative methods in evaluation research. California. USA: Bervely Hills.

Digital, C. D. (2018). PDF) Cuaderno Docentes Digital - 2018 - Digital. Available from: https://www.researchgate.net/ publication/332267557_Cuaderno_ Docentes_Digital_-_2018_-_Digital [accessed Apr 16 2020. Obtenido de https://www.researchgate.net/ publication/332267557

Edutopia. (2016). Resources for teaching growth mindset. Retrieved from http://www. edutopia.org/article/growth-mindsetresources MacMeekin, M. (. (s.f.). Edutopia. Obtenido de Resources 
for teaching growth mindset: https:// anethicalisland.wordpress.com/2013/0

Espinel, M., \& Llerena, G. (2013). La escritura en un proyecto de AAgroecologia. Textos de Didáctica de la Lengua y de la Literatura \#64, pp.56-68.

Feito, R. (2011). Los retos de la participación escolar. Elección, control y gestión de los centros educativos. Madrid, España: Morata. [ Links ]. Madrid, España: Morata. [ Links ]: Morata. [ Links ].

Fisher, C., \& Zimmerman, D. T. (2015). A Garden -Based Approach to Teaching Life Science Produces Shfts in Students'Attitudes toward the Environment Carley. (ISSN:1306-3065, Ed.) International Society of Educational Research, 10 (1),pp. 51-66.

Gonzales, J. ( 2015 ). Mejoramiento de la Producción escrita en inglés de grado sexto $b$ a partir de la implementación de un Taller de escritura creativa.

Gonzales, M. (2018). El huerto escolar como recurso didactico en educación Infantil.

Haros, B. G. (2013). Huerto escolar: estrategia educativa para la vida. Ra Ximhai. Revista de Sociedad, Cultura, Desarrollo, 9, 543-558. [ Links ]. Revista de Sociedad, Cultura, Desarrollo \#9, PP. 543-558.

Mejía, L. (2018). El huerto escolar como un epacio de aprendizaje para la enseñanza delcontenido reproducción en plantas en el grado séptimo. Obtenido de http://bdigital.unal.edu. co/6544171798575854.2018.pdf.ed

MEN. (2006). Estándares Básicos de Competencias en LenguaExtranjera :Inglés. . Series de Guís del MEN \#22.
Moreira M. A. y Greca, I. M. (2003). Cambio Conceptual: análisis crítico y propuestas a la luz de la Teoría del Aprendizaje Significativo. Ciência \& Educação, 9(2), 301-315.

Moreira, M. (s.f.). Aprendizajes Significativo como referente para la organización de la enseñanaza. Obtenido de Archivos de Ciencias de la educación, 11 (12).: https://doi.org/10.24215/23468866e029,

Morgan, P. W. (2010). The impact of nutrition education with and without a school garden on knowledge, vegetable intake and preferences and quality of school life among primary-school students. Public Health Nutrition,. pp 211 - 235.

Rivera M, J. L. (2004). El Aprendizaje significativo y la Evaluación de los Aprendizajes. Revista de Investigación Educativa, 8, 47-52.

Sántiz, G. (2018). El huerto escolar, oportunidad para fortalecer el trabajo colaborativo y la integración entre los estudiantes de escuelas primarias. . El Colegio de la Frontera Sur, . San Cristóbal de Las Casas, México. .

Savage. (2010). The context for cross -Curricular Teaching and Learning in the Secundary school. (R. T. group, Ed.) . Londres, New York: Series edited by School.

Scott, R. (2017). "Rethinking the environment", A Modern Guide to Rethinking Economics, . Cheltenham: . Rochon y S. Rossi (eds.), Cheltenham, Edward Elgar.

Steward, M. (s.f.). (U. d. primaria, Productor). (s.f.). Obtenido de Steward, M. (s.f.). http://repository.tcu.edu/ handle/116099117/4433. (U. d. primaria, Productor) 
Timmerman, V. (s.f.). Cross-curricular teaching: How does it work?

Vela, F. (2001). Un acto metodológico básico de la investigación social: la entrevista cualitativa. En M. L. Tarrés (Coord.), Observar, escuchar y comprender: Sobre la tradición cualitativa en la investigación social (pp. 63-92). México: FLACSO-El Colegio. México: FLACSO-El Colegio.

Williams, D. y. (2013). Impact of garden-based learning on academic outcomes in schools: Synthesis of research between 1990 and 2010. Review of Educational Research,, 211-235. doi:doi: $10.3102 / 0034654313475824$ 